\title{
Pandemic Risk Assessment Model (PRAM): a mathematical modeling approach to pandemic influenza planning
}

\author{
D. C. DOVER ${ }^{1 *}$, E. M. KIRWIN ${ }^{1}$, N. HERNANDEZ-CERON ${ }^{2}$ AND \\ K. A. NELSON ${ }^{3}$ \\ ${ }^{1}$ Analytics and Performance Reporting Branch, Alberta Health, Alberta, Canada \\ ${ }^{2}$ Department of Mathematics, Purdue University, IN, USA \\ ${ }^{3}$ Health Protection Branch, Alberta Health, Alberta, Canada
}

Received 17 April 2016; Final revision 7 July 2016; Accepted 28 July 2016;

first published online 22 August 2016

\section{SUMMARY}

The Pandemic Risk Assessment Model (PRAM) is a mathematical model developed to analyse two pandemic influenza control measures available to public health: antiviral treatment and immunization. PRAM is parameterized using surveillance data from Alberta, Canada during pandemic H1N1. Age structure and risk level are incorporated in the compartmental, deterministic model through a contact matrix. The model characterizes pandemic influenza scenarios by transmissibility and severity properties. Simulating a worst-case scenario similar to the 1918 pandemic with immediate stockpile release, antiviral demand is $20 \cdot 3 \%$ of the population. With concurrent, effective and timely immunization strategies, antiviral demand would be significantly less. PRAM will be useful in informing policy decisions such as the size of the Alberta antiviral stockpile and can contribute to other pandemic influenza planning activities and scenario analyses.

Key words: Influenza, mathematical modelling, pandemic, public health.

\section{INTRODUCTION}

Influenza is a viral respiratory illness that leads to cough, fevers, myalgia, headaches, and fatigue. In high-risk individuals, influenza can cause severe illness and death. Pandemic influenza is defined as an influenza 'epidemic occurring worldwide, or over a very wide area, crossing international boundaries and usually affecting a large number of people' [1]. Influenza pandemics occur when an influenza virus mutates so that it may be transmitted more easily

\footnotetext{
* Author for correspondence: Mr D. C. Dover, 23rd Floor, ATB Place North, 10025 Jasper Avenue, Edmonton, Alberta, T5J 1S6, Canada.

(Email: doug.dover@gov.ab.ca)
}

from person to person. When exposed to the new virus, many people may become ill as they will have little to no immunity.

The health impacts of pandemic influenza can be mitigated with public health interventions such as immunization, antivirals, hand hygiene and social distancing. The use of antivirals is twofold: as a treatment, or as a prophylactic measure for those who have come into close contact with a case of influenza. In 2004, Canada established the National Antiviral Stockpile (NAS) to secure a supply of antivirals in the event of pandemic influenza. The Canadian Antiviral Stockpile Management Task Group (CASMTG) recommends that each province purchase a stockpile sufficient to treat $17 \cdot 5 \%$ of their population [2]. Optimizing the size of a provincial 
antiviral stockpile is particularly complex, as it requires predicting the communicability and severity of a pandemic influenza as well as the subsequent effect on antiviral demand.

Mathematical models provide an opportunity to evaluate the potential impacts of decisions and help to plan for efficient use of public resources. Models simulating influenza pandemics and mitigation strategies in the literature follow one of three broad structures: (i) static Markov chain [3-9], (ii) deterministic compartmental (with stochastic elements in some cases) [5, 6, 10-30], and (iii) network or individual/agent-based stochastic [31-43]. Within these categories, which themselves are quite fluid, there is a great deal of diversity. Multiple waves may be modelled by defining populations as open or closed, forcing seasonality by seeding infected individuals, manipulating the force of infection, or by simulating the spread of multiple influenza strains. Many mitigation strategies can be modelled and compared; immunization, antiviral treatment (varying formulation with changing viral resistance) and social distancing (full quarantine, school, workplace and medical facility closure, travel restrictions). Each model structural type has merits for different purposes.

In this paper, we have developed a deterministic, compartmental model. The Pandemic Risk Assessment Model (PRAM) was developed to produce outcome measures that are familiar to public health officials: the number of persons medically attended, hospitalized, and dying due to pandemic influenza. Interventions of both immunization and antiviral treatment are modelled, allowing for targeting and delays in the release of each. The age and risk group structure in PRAM was inspired by other Canadian models [18, 27, 30].

In the following sections we describe the development of PRAM, its attributes, how the model parameters interact, results, and data required to customize the model to various jurisdictions.

\section{METHODS}

To model pandemic influenza in Alberta, PRAM was developed and programmed in Mathematica ${ }^{\circledR}$ (http:// www.wolfram.com/). Data and parameters from the province of Alberta, Canada were used. The model was fit to Alberta outcomes in terms of antivirals delivered, total medically attended and hospitalized, and total deaths from the H1N1 pandemic in $2009 \mathrm{~A}(\mathrm{H} 1 \mathrm{~N} 1) \mathrm{pdm} 09$.

Generating visual output and allowing for intuitive scenario analysis by public health officials are important components of the model dissemination process. PRAM allows users to simulate different pandemic influenza scenarios and generate visual output with no need for programming. The model can be distributed as a computable document format (CDF) file to allow use of PRAM without requiring a license for Mathematica, addressing the call for models that can 'be downloaded and synthesized using personal computers' raised by Pretio et al. [44].

\section{Model type selection}

PRAM is a deterministic compartmental model. This model type was selected because a static Markov model would not fully capture the dynamics of influenza in the population, misestimating the dynamic impacts of immunization and antiviral use. While an agent-based model would provide an accurate and detailed simulation of a pandemic, these models are highly sensitive to the details of interpersonal contact [38] and we are not confident that appropriate data is available or could be estimated for Alberta or Canada. In addition, deterministic models provide similar outcomes to agent-based models [43]. Further, deterministic compartmental models are more easily understood by policy-makers because a single result is given consistently and rapidly relative to agentbased models.

\section{Transmissibility-severity framework}

During H1N1, the experience across public health departments was that the simple descriptions of a pandemic (using only $R_{0}$ ) were insufficient. Details on how transmissible and severe the influenza virus is are key factors influencing public health actions. This paradigm shift has been recognized in recent frameworks $[45,46]$ and changes in the WHO pandemic criteria [47]. Transmissibility measures the contagiousness or infectiousness of a virus, while severity captures the degree of symptoms and level of care required for infected individuals. We introduce an explicit mathematical representation of transmissibility and severity in PRAM. The severity and transmissibility parameters can be altered by users to simulate pandemic influenza scenarios and provide an intuitive characterization of pandemic influenza which is both graphical and numerical.

The transmissibility parameter influences the probability of virus transmission to a susceptible person from an infectious person. In form, it is similar to the 
proportionality factor discussed in Ogunjimi et al. [48]. This separates the contact matrix and transmissibility parameter ensures the model can be easily adapted to any local context. Under this specification, the reproductive factor $\left(R_{0}\right)$ is a function of both the viral characteristics (transmissibility) and local behaviours (contact matrix).

The severity parameter uniquely characterizes the increased health risk associated with a particular pandemic virus and can be varied. It affects (i) the likelihood of being medically attended (given infection); (ii) the likelihood of hospitalization; and (iii) the likelihood of death. The severity parameter is implemented as a common odds ratio to each of these transitions, and is anchored at 1.0 in the $\mathrm{A}(\mathrm{H} 1 \mathrm{~N} 1) \mathrm{pdm} 09$ scenario.

\section{Policy levers}

PRAM was designed to allow users to examine the effect of different policies on outcomes. The antiviral policy lever allows the user to specify the date on which antivirals are distributed, and whether they are distributed to only those at high risk, only those at low risk, both, or not distributed. A delay in the release of the antiviral stockpile may also be specified in the model.

The vaccine policy lever controls when specific age-risk groups are eligible to be immunized. Age-risk groups can be allocated to two stages of vaccine release. The 14 age-risk groups (seven age groups and two risk groups) are allocated to one of the two stages, each with their own delay in vaccine release. Age-risk groups specified as part of the first stage can receive vaccine [i.e. transition from susceptible (1) to vaccinated (3) or vaccine failure (4)] on or after the first stage delay. All age-risk combinations can receive vaccine after the second stage delay. Vaccination volume is capped at a maximum volume of daily doses administered, representing a real limitation in the healthcare delivery system. In Alberta, influenza immunization is publicly provided in a onedose format, with the exception of infants who receive two doses. PRAM makes the simplifying assumption of a universal one-dose schedule.

For every scenario, the user inputs antiviral and vaccine availability as a proportion of the total population.

\section{Model structure}

The model structure was based upon the formulation used in the Tuite et al. [27, 30] and Greer \& Schanzer
[18] models. We preserved the seven age and two risk groups used in their model, and divided the broader categories (Susceptible, Exposed, Infectious, Recovered) into sub-compartments specified to be interpretable and meaningful to policy-makers.

The Alberta population ( $n=3617411)$ as of 1 July 2009 was divided into the same seven age groups $(0-4$, $5-14,15-19,20-24,25-54,55-64, \geqslant 65$ years) as those used in other Canadian models. To simulate mixing between these age groups, a contact matrix from Finland [49] was modified to reflect the age groups used in PRAM.

The population in each age group was further subdivided into high- and low-risk groups, defined to capture those with an increased risk of an adverse outcome (medical attendance, hospitalization, death). High-risk individuals were identified in the Alberta population as those with a diagnosis of asthma, diabetes, renal or kidney disease, hypertension, chronic obstructive pulmonary disease and congestive heart failure.

The model assumes the population is allocated between the mutually exclusive health states denoted in Figure 1. Individuals typically start in either the susceptible (1) or susceptible, non-immunizer (2) compartments. This separation of immunizers and non-immunizers recognizes that a subset of any given population will not immunize. Individuals from the susceptible compartment (1) may be vaccinated (3), which if successful will move them to the recovered/immune compartment (12) after a holding period to reflect a mounting immune response (during which they may still become exposed to the virus and infectious) or the immunization can fail, moving them to the vaccine failure compartment (4). Individuals from compartments (1)-(4) (outlined in blue) may be exposed to the virus, and can move to the exposed (5) compartment. These individuals will develop infections that will or will not require medical attention, dependent on the severity of the virus and their risk status, moving either into the medically attended compartment (6), or the not medically attended compartment (7). Persons who are exposed to an infectious person but do not become infected are not included in this category. Individuals in the not medically attended compartment (7) are assumed to recover to the recovered/immune compartment (12) at a fixed rate, with no further complications.

The infectious compartments (6)-(10) are initially divided between medically attended (6) and not medically attended (7). The choice to use the term 


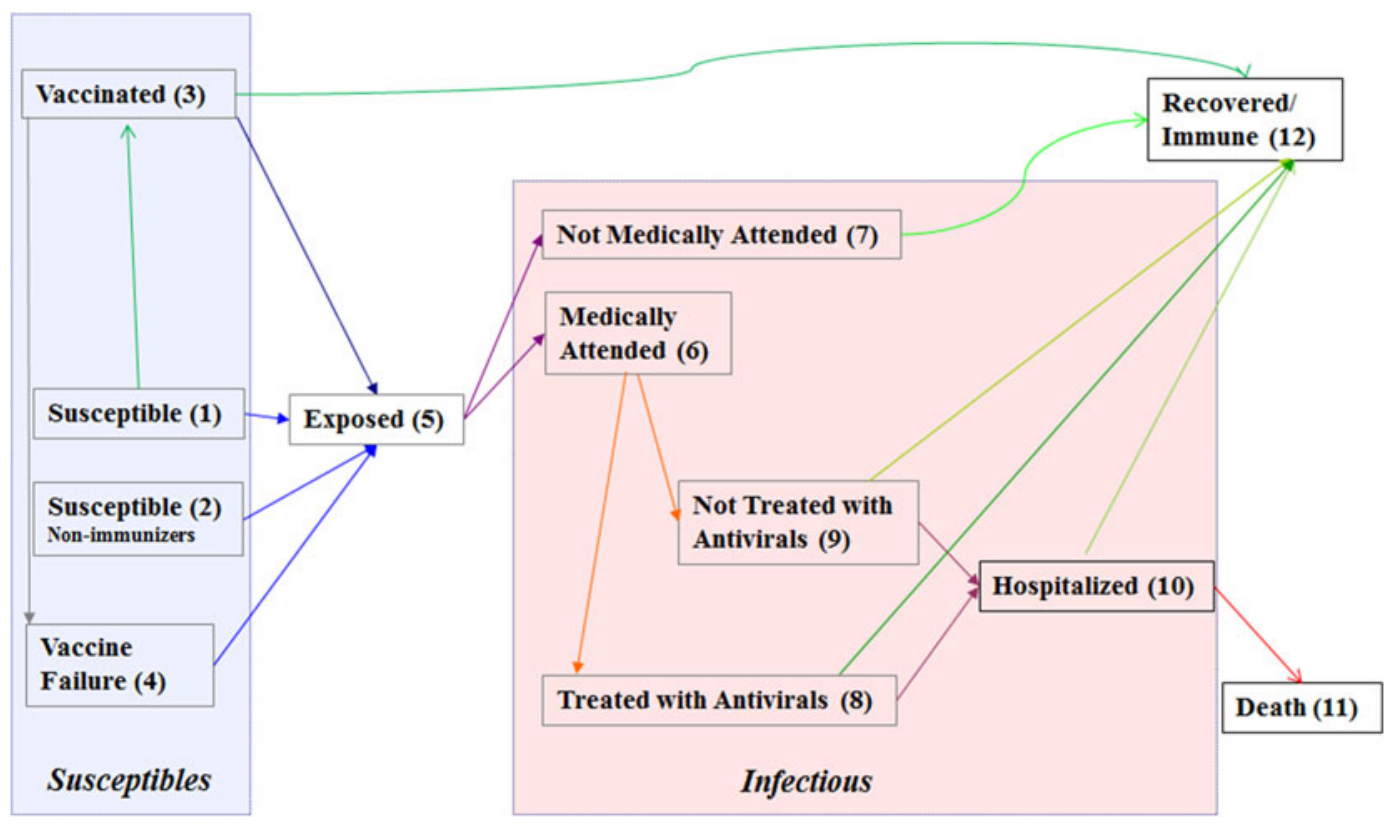

Fig. 1. Alberta Pandemic Risk Assessment Model structure.

'medically attended' to describe the infectious compartments (as opposed to, for example, 'symptomatic') was selected because being symptomatic is not easily measured by surveillance data, and further, does not necessarily imply burden on or interaction with the healthcare system, whereas data on health system interactions are likely to be readily available. Population-based administrative data is available for those medically attended during H1N1 in Alberta, while information regarding the proportion of ill persons who were symptomatic is highly variable. Appeals to the broader literature found widely varying estimates of the proportion symptomatic $[50,51]$ in addition to others cited in a systemic review and meta-analysis [52]. For the purposes of PRAM, medically attended is defined as primarily a visit to a primary-care physician, but could include an emergency-department visit or initial contact with the healthcare system. We find this definition has a clear meaning with direct implications for assessing burden on the health system.

Depending on the antiviral policy scenario modelled, medically attended (6) individuals may move either to the treated with antivirals compartment (8), or the not treated with antivirals compartment (9). We assume full adherence of individuals treated with antivirals. From either of these two compartments, individuals may be hospitalized (10), or move to the recovered/immune compartment (12); if they were treated with antivirals, the antiviral effectiveness parameter affects these transitions. Those hospitalized (10) will either move into the death (11) or recovered/ immune (12) compartments. We assume that any individuals in the hospitalized compartment (10) are treated with antivirals, even though they may have already been treated in compartment (8). Individuals in the treated with antivirals (8) or hospitalized (10) compartments are assumed to contribute less to the force of infection in the model, as their transmissibility has been attenuated by antiviral treatment, reflected in the force of infection equation given in the Supplementary material. The antiviral treatment effectiveness of individuals entering the hospitalized compartment (10) from the treated with antivirals compartment (8) is assumed to be zero. The probability of being medically attended (6), hospitalized (10) and death (11) are all functions of both the severity parameter and of the risk group.

In the initial state for the baseline model, all individuals are allocated between the susceptible (1) and susceptible, non-immunizers (2) compartments. For the results presented here, no prior immunity is assumed. To capture prior immunity, individuals could also be initially allocated to the recovered/immune compartment (12).

\section{Parameter values}

Alberta Ministry of Health surveillance data from the 2009 H1N1 pandemic was leveraged for model parameters. Where this was not possible, values from 
Table 1. Model fitting

\begin{tabular}{llll}
\hline \hline & Observed value & Fitted value & Relative error \\
\hline Medically attended & 129619 [Alberta Health Internal Data] & 123109 & $5 \cdot 0 \%$ \\
Antivirals distributed & 45721 [Alberta Health Internal Data] & 48500 & $6 \cdot 1 \%$ \\
Hospitalized & 1276 [56] & 1244 & $2 \cdot 5 \%$ \\
Deceased & $71[56]$ & 70 & $1 \cdot 4 \%$ \\
\hline \hline
\end{tabular}

Canadian or international literature were used. The Supplementary material contains the rationale and sources, and a discussion of the parameter values used in the model.

\section{Model fitting}

Three parameters - the probability of transitioning to medically attended, hospitalized, and death - were fit simultaneously using weighted least squares to the total counts from the $2009 \mathrm{H} 1 \mathrm{~N} 1$ pandemic experience in Alberta, as well as the antivirals distributed For the purposes of model fitting, no vaccine distribution was assumed since vaccine release in Alberta occurred near or after the peak [47]. Observed and fitted counts for those medically attended, hospitalized, and dying are given in Table 1.

\section{Basic reproductive number}

The basic reproductive number $\left(R_{0}\right)$ can be interpreted as the number of cases caused by each incident case. In our model, $R_{0}$ is a function of the transmissibility and severity parameters and of the contact matrix, so the value of $R_{0}$ varies as transmissibility and severity are altered. $R_{0}$ is computed analytically and reported in the model assuming no intervention (e.g. no antiviral or immunization interventions). Using the next-generation approach [53, 54] (a standard mathematical method to analyse epidemic models), a simple formula to compute $R_{0}$ is given (calculation details are given in the Supplementary material).

\section{Scenarios}

PRAM can be used to explore any number of possible scenarios for pandemic influenza planning. We focus on two scenarios for the purposes of exploring the effects on intervention options: the baseline H1N1 case and a worst-case scenario.
In the baseline case of 2009 pandemic H1N1 (severity $1 \cdot 0$, transmissibility $2 \cdot 5 \%$ ), combined impacts of immunization and antiviral interventions are explored. The initial output corresponds to the observed influenza $\mathrm{A}(\mathrm{H} 1 \mathrm{~N} 1) \mathrm{pdm} 09$ experience in Alberta. Various interventions (immunization and antivirals, varying timing of release and target population) are explored.

For the worst-case scenario, transmissibility and severity were set to $3 \cdot 5 \%$ and $7 \cdot 5$, respectively, producing morbidity and mortality to correspond to the 1918 influenza pandemic. To calculate the maximum antiviral demand, no immunization is distributed and the antiviral stockpile is released without delay. Because the model was designed to inform choices related to the size of the NAS, the outcome of interest for each scenario was the total number of antiviral doses demanded as a proportion of the total population. The associated burden is quantified by the number medically attended, hospitalized, and dying in each scenario.

As a sensitivity analysis across different pandemic influenza scenarios, we explore antiviral use and hospitalizations for a range of transmissibility and severity parameter values (of $2 \cdot 5-5 \cdot 0 \%$ by $0 \cdot 2 \%$ increments, and $1-10$ by increments of 1 , respectively) with no vaccine release and immediate antiviral release to both high and low risk groups.

\section{RESULTS}

Across the range of policy options evaluated for a relatively mild pandemic, $\mathrm{A}(\mathrm{H} 1 \mathrm{~N} 1) \mathrm{pdm} 09$, the maximum antiviral demand of $3 \cdot 2 \%$ of the current stockpile occurred when there was no vaccine available and the stockpile was released immediately. In the high-demand scenario, antiviral demand reached $20 \cdot 3 \%$ of the population. The antiviral demand and associated outcomes are given in Tables 2 and 3 .

The joint influence of severity and transmissibility on antiviral demand and the number of hospitalizations is seen in Figures 2 and 3. 
Table 2. Extended model output: H1N1 outcomes

\begin{tabular}{|c|c|c|c|c|c|c|c|c|}
\hline Scenario & $\begin{array}{l}\text { Antiviral } \\
\text { release policy }\end{array}$ & $\begin{array}{l}\text { Antiviral } \\
\text { release delay }\end{array}$ & Vaccine & $\begin{array}{l}\text { Total antiviral } \\
\text { required } \\
\text { (\% stockpile) }\end{array}$ & $\begin{array}{l}\text { Total vaccine } \\
\text { required } \\
\text { (\% pop.) }\end{array}$ & $\begin{array}{l}\text { Total } \\
\text { exposed* }(\%)\end{array}$ & $\begin{array}{l}\text { Total } \\
\text { hospitalized }\end{array}$ & $\begin{array}{l}\text { Total } \\
\text { deaths }\end{array}$ \\
\hline \multicolumn{9}{|l|}{ Base case } \\
\hline & None & & None & 0 & 0 & $44 \cdot 4$ & 1697 & 95 \\
\hline \multicolumn{9}{|c|}{ Antiviral effects } \\
\hline & High risk & Peak & None & $1 \cdot 0$ & 0 & $43 \cdot 6$ & 1275 & 71 \\
\hline & Both & Peak & None & $1 \cdot 3$ & 0 & $43 \cdot 3$ & 1244 & 70 \\
\hline & High risk & Immediate & None & $2 \cdot 3$ & 0 & $42 \cdot 6$ & 688 & 37 \\
\hline & Both & Immediate & None & $3 \cdot 2$ & 0 & $41 \cdot 8$ & 612 & 34 \\
\hline \multicolumn{9}{|c|}{ Vaccine effects } \\
\hline & None & & Peak & 0 & $18 \cdot 1$ & $40 \cdot 6$ & 1542 & 86 \\
\hline & None & & Immediate & 0 & $23 \cdot 9$ & $24 \cdot 8$ & 920 & 51 \\
\hline \multicolumn{9}{|c|}{ Combined effects } \\
\hline & Both & Peak & Peak & $1 \cdot 1$ & $18 \cdot 2$ & $39 \cdot 9$ & 1192 & 67 \\
\hline & Both & Immediate & Peak & $2 \cdot 7$ & $19 \cdot 8$ & $35 \cdot 8$ & 520 & 29 \\
\hline & Both & Peak & Immediate & $1 \cdot 1$ & $23 \cdot 9$ & $22 \cdot 7$ & 521 & 29 \\
\hline & Both & Immediate & Immediate & $1 \cdot 6$ & $23 \cdot 9$ & $21 \cdot 1$ & 300 & 17 \\
\hline
\end{tabular}

* 'Total exposed' is defined as the cumulative proportion of the population exposed to the virus in the exposed compartment (5) who will go on to be medically attended (6) or not medically attended (7). It does not refer to persons in the vicinity of an infectious person who does not become infected.

Table 3. High antiviral demand scenario 1918

\begin{tabular}{|c|c|c|c|c|c|c|}
\hline Scenario & $\begin{array}{l}\text { Antiviral target } \\
\text { population }\end{array}$ & Vaccine & $\begin{array}{l}\text { Total antivirals } \\
\text { demanded }(\%)\end{array}$ & $\begin{array}{l}\text { Total exposed } \\
\text { (\% pop.) }\end{array}$ & $\begin{array}{l}\text { Total } \\
\text { hospitalized }\end{array}$ & $\begin{array}{l}\text { Total } \\
\text { deaths }\end{array}$ \\
\hline 1918 & High risk & None & $12 \cdot 0$ & $75 \cdot 0$ & 26885 & 7308 \\
\hline 1918 & Both & None & $20 \cdot 3$ & $74 \cdot 9$ & 21248 & 6182 \\
\hline
\end{tabular}

\section{DISCUSSION}

\section{PRAM}

PRAM was developed to address the needs of Alberta's decision-makers, with a novel parameterization of transmissibility and severity, and was specifically designed using data from public health surveillance for preparedness planning. The model is able to replicate several past influenza pandemics experienced in Alberta in a visual and easily understood implementation.

$\mathrm{A}(\mathrm{H} 1 \mathrm{~N} 1) \mathrm{pdm} 09$ was used as the reference case for modelling other influenza pandemics. Worse scenarios may be simulated by higher transmissibility and severity choices. Adjusting the severity and transmissibility parameters of PRAM to replicate the Spanish Flu of 1918 as a worse-case scenario, the total antiviral demand will slightly exceed the current CASMTG recommendation of a stockpile size sufficient to treat $17 \cdot 5 \%$ of the population.
This finding has important policy implications, as decisions regarding the size of a stockpile must be made without knowledge of the timing and severity of the next pandemic. Decision-makers can consider model outputs in weighing the risks and benefits of maintaining different stockpile sizes.

\section{Comparisons to similar models}

The findings of PRAM are consistent with similar models. In a model examining a treatment- only antiviral strategy with no vaccination, Gani et al. [29] found that an $R_{0}$ of 1.39 [corresponding closely to an $R_{0}$ of 1.41 from our $\mathrm{A}(\mathrm{H} 1 \mathrm{~N} 1) \mathrm{pdm} 09$ scenario] would require a stockpile sufficient to treat $12 \%$ of the population. PRAM requires a $3 \cdot 2 \%$ stockpile under a similar scenario. Reductions in hospitalizations are similar between the two models: $77 \%$ in Gani et al. and 64\% in PRAM. Differences in stockpile requirements may be due to a considerably denser 


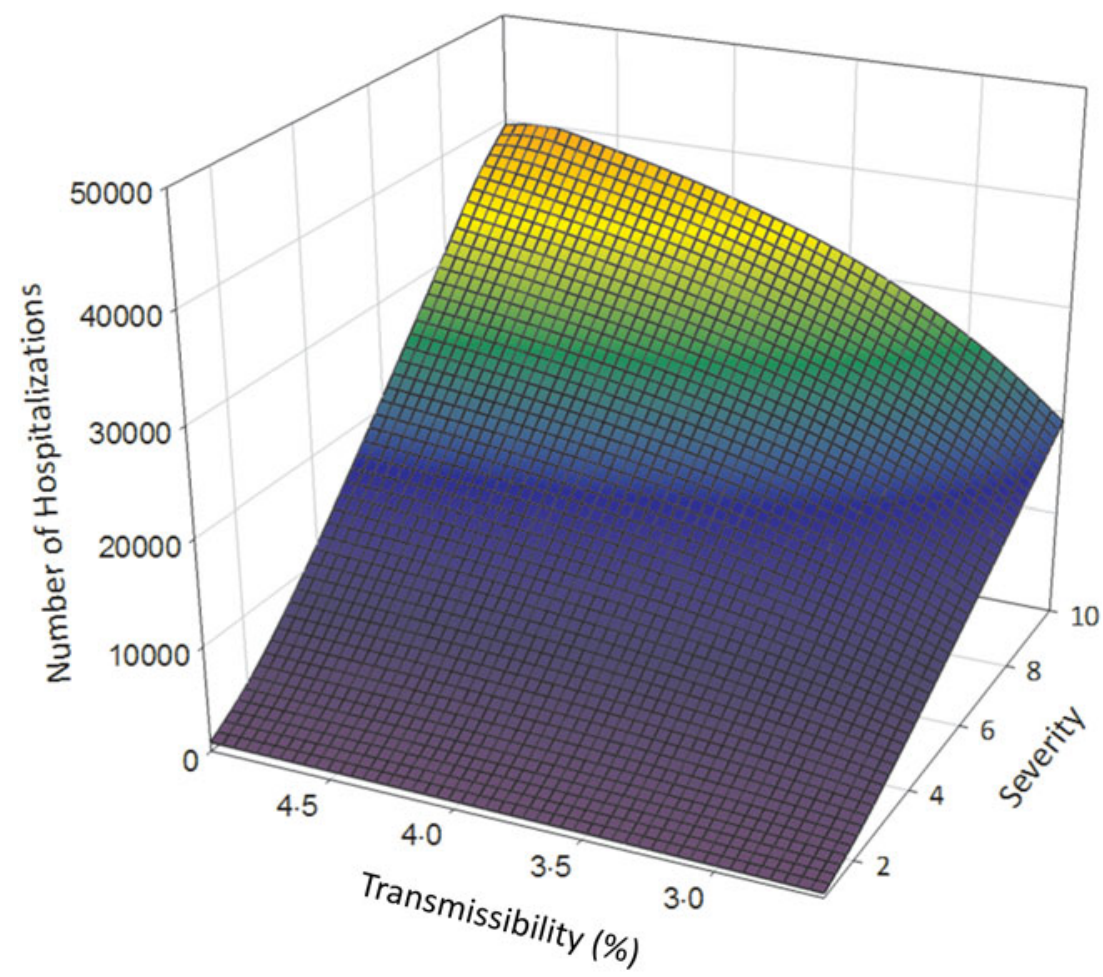

Fig. 2. Impact of severity and transmissibility on hospitalizations.

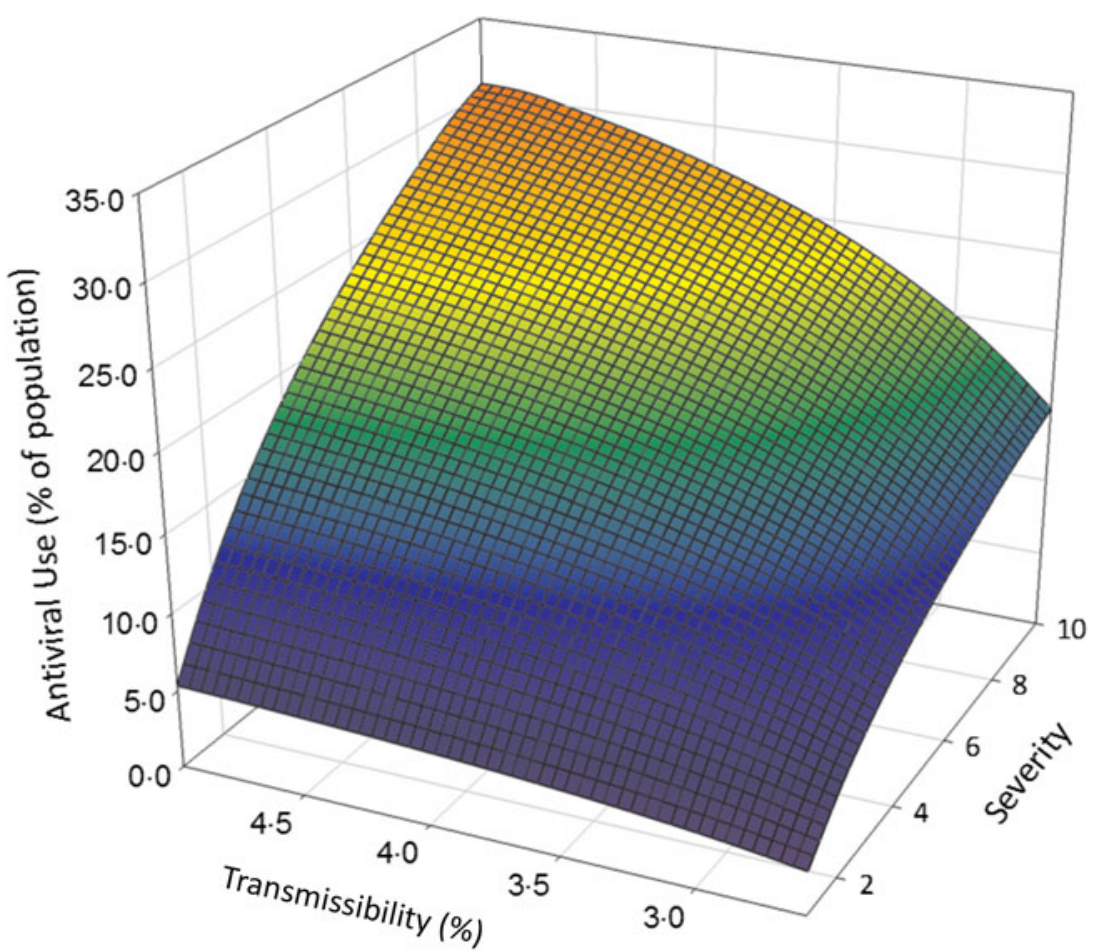

Fig. 3. Impact of severity and transmissibility on antiviral use. 
population and the assumption of homogenous mixing in [29].

Using PRAM to simulate a scenario similar to the $1.6 R_{0}$ modelled in Greer \& Schanzer [18], with immediate stockpile release, and vaccine distribution after 3 months, antiviral demand in PRAM (severity 2, transmissibility $2 \cdot 85 \%$ ) is $4.3 \%$. This corresponds to the range for similar scenarios modeled by Greer \& Schanzer, where antiviral treatment requirements to treat only symptomatic pandemic influenza cases range in value from $3.9 \%$ to $5 \cdot 4 \%$. Greer \& Schanzer's estimation of antiviral wastage due to treatment of influenza-like illness (ILI) cases increase this to a total stockpile requirement of $5 \cdot 4-96 \cdot 4 \%$. Key differences exist between PRAM and [18] in the assumptions for care-seeking and antiviral treatment. Greer \& Schanzer assume that $60 \%$ of those who were infected will be symptomatic, that $50-70 \%$ of those who are symptomatically infected will receive antiviral treatment, and that individuals have three episodes of ILI annually, which require antiviral treatment during pandemic influenza. These assumptions are quite different from PRAM, where infected individuals are care-seeking or not (based on empirical data and modified by the severity parameter). When individuals present for care, they are uniformly dispensed antivirals or not based on the policy levers and antiviral availability, thus, the parameterization of [18] guarantees that between $30 \%$ and $42 \%$ of those infected will receive antiviral treatment for pandemic influenza in addition to ongoing treatment for ILI, while PRAM has a distribution that varies with time, risk group, and strain severity, but will not account for stockpile depletion outside of the policy parameters (prescriptions for patients who do not have influenza and other wastage). Both PRAM and [18] ignore misdiagnosis, where laboratory-confirmed influenza cases are misdiagnosed clinically. In PRAM, the assumption that the proportion misdiagnosed remains constant over time is made. This is justifiable because the model is fitted to surveillance data that will contain some proportion of misdiagnosed cases, which would also represent a real burden in model predictions. Miller et al. [55] show that 44 $71 \%$ of influenza cases may be misdiagnosed, which could greatly reduce the number of antiviral prescriptions issued. However, during a pandemic, diagnostic behaviour is likely to change, and antivirals may be prescribed to cases which are not clinically diagnosed. Other work considering antiviral demand varied the proportion of wastage in sensitivity analyses [9], while others used a decay function to model stockpile attrition [42].

PRAM also differs from the existing literature of compartmental deterministic models evaluating the use of both antivirals and vaccine $[6,18,23]$ in the treatment of asymptotic infectious individuals, and the mechanisms governing either the release of an antiviral stockpile or the implementation of an immunization programme.

\section{Model limitations}

Mathematical modelling relies upon simplifying assumptions so that a complex system can be reduced to a feasible model with parameters that can be both measured and justified. These assumptions result in limitations with implications for how policy-makers must interpret model outputs. For example, to be admitted to a hospital, it is assumed that an individual has first been 'medically attended', meaning that they have interacted with a primary-care physician or similar. While this may not always be the case, it is a justifiable simplification of reality. PRAM is designed such that the 'total antiviral required' output measures the total demand for antiviral prescriptions issued, in both private clinics and in hospitals. The number of prescriptions issued must be translated into individual treatment courses of multiple doses which will vary based on patient age and other characteristics. In addition, because PRAM does not include wastage, it is crucial that decision makers consider their local context in allocating additional units for wastage. Similarly, PRAM assumes perfect adherence for those issued prescriptions for antivirals, and does not evaluate distribution restrictions on antivirals. Users could estimate non-perfect adherence by multiplying some factor through the output, but this would not capture a more sustained epidemic curve.

Similar considerations affect the interpretation of vaccine usage. In Alberta, influenza is given as a single-dose programme, so that the 'total number immunized' reflects the total number of doses required, with the exception of infants requiring two doses. In jurisdictions with a multi-dose schedule, the 'total number immunized' should be adjusted to reflect total doses required.

Other considerations in the interpretation of model output are the expected levels of misdiagnosis (failure to correctly diagnose influenza when it is present and incorrect diagnosis of influenza when it is not present), prescriptions issued outside of the stockpile release, 
and prescriptions not completed. PRAM does not account for age or risk-based variation in vaccine and antiviral effectiveness. However, the degree to which these will vary is not often known prior to a pandemic, so any effort to differentiate by group may confer a false sense of accuracy.

PRAM does not evaluate other public health interventions such as public health messaging for hand hygiene and social distancing. Changing antiviral resistance is not considered within a simulation, but the antiviral effectiveness parameter could be adjusted to reflect resistance.

Deterministic models like PRAM assume a simplified flow through the healthcare system. For example, the only way to be hospitalized in PRAM is through the medically attended compartment. While this assumption is empirically supported and sensible from a planning perspective, those interpreting results should be aware of it. Referring to Table $1,<1 \%$ of those medically attended become hospitalized, and only a proportion of those would have been admitted directly to a hospital without a prior physician visit. In this way, this simplifying assumption does not greatly influence model outcomes.

PRAM assumes a single pandemic wave. The assumption comes from the underlying choice to incorporate only a fixed contact matrix, a closed population, and a fixed transmissibility parameter. While initial infected individuals could come from outside of Alberta, there is no introduction of exogenous infected individuals beyond the initial allocation. While PRAM could be adjusted to include a variable transmissibility parameter, a central concept to the model was the characterization of a pandemic strain by severity and transmissibility. These assumptions are reasonable for pandemic preparedness planning where planning revolves around an unexpected event in a relatively short time-frame.

PRAM outputs, like all mathematical models, rely upon the parameters used as inputs. While many of the parameter choices have been discussed previously, disentangling the contact matrix, transmissibility and severity is novel to PRAM. In particular, estimates of transmissibility are rare. Our use of a household contact study, while specific to Alberta during H1N1, may under- or over-estimate the transmissibility parameter (as the infectivity period for influenza is less than the 2 weeks of exposure assumed, yet a contact-day within a household is likely longer than a non-household contact-day). Similarly, estimates of severity, as they pertain to the transitions in the model, are rarely published but often available from administrative health data sources. For PRAM and newer models describing pandemic influenza with both of the severity and transmissibility dimensions, these concepts and estimates of their parameters require continued research.

PRAM provides a useful tool for public health professionals to assess the potential impacts of pandemic influenza. The model assumptions and associated limitations must be borne in mind whenever outcomes are interpreted.

\section{The use of PRAM in public policy}

Pandemic influenza planning often utilizes multiple scenarios to strategize for unknown future influenza pandemics. PRAM allows users to adjust the transmissibility and severity parameters, generating new scenarios to simulate outcomes such as the number of people medically attended, hospitalized or deceased using Alberta's population. These outcomes for each of these states allow planners to assess the demand on specific healthcare resources.

Policy-makers can adjust the policy levers to simulate the outcomes of a particular policy decision within each of these scenarios. For example, by specifying how many days into a pandemic the antiviral stockpile will be released, the model will simulate the change in outcomes in Alberta's population for that scenario. Further, the user could then adjust the scenario to find out the simulated effect of releasing these antivirals to only high-risk groups. This flexibility allows the user to assess the potential effects and interactions of policy options on the Alberta population and how those policies may complement or conflict with each other.

\section{CONCLUSION}

PRAM can assist in informing policy and programme decisions. The model compartments are chosen to capture elements both meaningful to decision makers, and the transmissibility-severity parameterization links clearly with identifiable and easily interpreted risk scenarios. The presentation of scenario options through an interactive, visual user interface allows non-technical professionals to compare multiple scenarios. The software is available from the authors upon request and as Supplementary material.

\section{SUPPLEMENTARY MATERIAL}

For supplementary material accompanying this paper visit http://dx.doi.org/10.1017/S0950268816001850. 


\section{ACKNOWLEDGEMENTS}

The authors thank the reviewers for providing helpful and insightful feedback greatly improving the manuscript.

\section{DECLARATION OF INTEREST}

None.

\section{REFERENCES}

1. Last JM. A Dictionary of Epidemiology, 4th edn. New York: Oxford University Press; 2001.

2. Public Health Agency of Canada. The Management of the National Antiviral Stockpile: Options Report (http://www.phac-aspc.gc.ca/alert-alerte/h1n1/antivir/indexeng.php). Accessed 6 February 2015.

3. Biggerstaff M, et al. Estimating the potential effects of a vaccine program against an emerging influenza pandemic-United States. Clinical Infectious Diseases 2015; 60: S20-S29.

4. Doyle A, et al. Influenza pandemic preparedness in France: modelling the impact of interventions. Journal of Epidemiology and Community Health 2006; 60: 399-404.

5. Khazeni $\mathbf{N}$, et al. Effectiveness and cost-effectiveness of expanded antiviral prophylaxis and adjuvanted vaccination strategies for an influenza A (H5N1) pandemic. Annals of Internal Medicine 2009; 151: 840-853.

6. Khazeni N, et al. Effectiveness and cost-effectiveness of vaccination against pandemic influenza (H1N1) 2009. Annals of Internal Medicine 2009; 151: 829-839.

7. O'Hagan JJ, et al. Estimating the United States demand for influenza antivirals and the effect on severe influenza disease during a potential pandemic. Clinical Infectious Diseases 2015; 60: S30-S41.

8. van Genugten ML, Heijnen MLA, Jager JC. Pandemic influenza and healthcare demand in the Netherlands: scenario analysis. Emerging Infectious Diseases 2003; 9: $531-538$.

9. Siddiqui M, Edmunds W. Cost-effectiveness of antiviral stockpiling and near-patient testing for potential influenza pandemic. Emerging Infectious Diseases 2008; 14: 267-274.

10. Alexander ME, et al. Emergence of drug resistance: implications for antiviral control of pandemic influenza. Proceedings of the Royal Society of London B: Biological Sciences 2007; 274: 1675-1684.

11. Alexander ME, et al. A delay differential model for pandemic influenza with antiviral treatment. Bulletin of mathematical biology 2008; 70: 382-397.

12. Arino J, Bowman CS, Moghadas SM. Antiviral resistance during pandemic influenza: implications for stockpiling and drug use. BMC Infectious Diseases. Published online: 22 Janurary 2009. doi: 10.1186/1471-2334-9-8.

13. Baguelin $\mathbf{M}$, et al. Vaccination against pandemic influenza $\mathrm{A} / \mathrm{H} 1 \mathrm{~N} 1 \mathrm{v}$ in England: a real-time economic evaluation. Vaccine 2010; 28: 2370-2384.

14. Bolton KJ, et al. Prior population immunity reduces the expected impact of CTL-inducing vaccines for pandemic influenza control. PLOS ONE. Published online: 26 March 2015. doi: 10.1371/journal.pone. 0120138.

15. Chowell G, et al. Adaptive vaccination strategies to mitigate pandemic influenza: Mexico as a case study. PLoS ONE. Published online: 3 December 2009. doi:10.1371/ journal.pone.0008164.

16. Fung ICH, et al. Modeling the Effect of School Closures in a Pandemic Scenario: Exploring Two Different Contact Matrices. Clinical Infectious Diseases 2015; 60: S58-S63.

17. Ferguson NM, et al. A population-dynamic model for evaluating the potential spread of drug-resistant influenza virus infections during community-based use of antivirals. Journal of Antimicrobial Chemotherapy 2003; 51: 977-990.

18. Greer AL, Schanzer D. Using a dynamic model to consider optimal antiviral stockpile size in the face of pandemic influenza uncertainty. PLoS ONE. Published online: 21 June 2013. doi: 10.1371/journal.pone.0067253.

19. Hansen E, et al. Strategies for the use of oseltamivir and zanamivir during pandemic outbreaks. Canadian Journal of Infectious Diseases \& Medical Microbiology 2010; 21: e28-e63.

20. Lee S, Chowell G, Castillo-Chávez C. Optimal control for pandemic influenza: the role of limited antiviral treatment and isolation. Journal of Theoretical Biology 2010; 265: 136-150.

21. Lipsitch M, et al. Antiviral resistance and the control of pandemic influenza. PLoS Medicine 2007; 4: 111-121.

22. McCaw JM, McVernon J. Prophylaxis or treatment? Optimal use of an antiviral stockpile during an influenza pandemic. Mathematical Biosciences 2007; 209: 336-360.

23. Nuno M, Chowell G, Gumel AB. Assessing the role of basic control measures, antivirals and vaccine in curtailing pandemic influenza: scenarios for the US, UK and the Netherlands. Journal of the Royal Society Interface 2007; 4: 505-521.

24. Prosper O, et al. Modeling control strategies for concurrent epidemics of seasonal and pandemic $\mathrm{H} 1 \mathrm{~N} 1$ influenza. Mathematical Biosciences and Engineering 2011; 8: 141-170.

25. Regoes RR, Bonhoeffer S. Emergence of drug-resistant influenza virus: population dynamical considerations. Science 2006; 312: 389-391.

26. Stilianakis NI, Perelson AS, Hayden FG. Emergence of drug resistance during an influenza epidemic: insights from a mathematical model. Journal of Infectious Diseases 1998; 177: 863-873.

27. Tuite AR, et al. Optimal pandemic influenza vaccine allocation strategies for the Canadian population. PLoS ONE. Published online: 12 April 2010. doi:10.1371/ journal.pone.0010520.

28. Zhang Q, Wang D. Antiviral prophylaxis and isolation for the control of pandemic influenza. International Journal of Environmental Research and Public Health 2014; 11: 7690-7712.

29. Gani R, et al. Potential impact of antiviral drug use during influenza pandemic. Emerging Infectious Diseases 2005; 11: 1355-1362.

30. Tuite A, et al. Seasonal vaccine allocation in the Canadian population during a pandemic. PLoS 
Currents: Influenza. Published online: 11 December 2009. doi:10.1371/currents.RRN1143.

31. Bansal S, et al. The shifting demographic landscape of pandemic influenza. PLoS ONE. Published online: 26 February 2010. doi:10.1371/journal.pone.0009360.

32. Germann TC, et al. Mitigation strategies for pandemic influenza in the United States. Proceedings of the National Academy of Sciences USA 2006; 103: 5935-5940.

33. Glass RJ, et al. Targeted social distancing design for pandemic influenza. Emerging Infectious Diseases 2006; 12: 1671-1681.

34. Halloran ME, et al. Modeling targeted layered containment of an influenza pandemic in the United States. Proceedings of the National Academy of Sciences USA 2008; 105: 4639-4644.

35. Kramer SC, Bansal S. Assessing the use of antiviral treatment to control influenza. Epidemiology and Infection 2015; 143: 1621-1631.

36. Longini IM, et al. Containing pandemic influenza with antiviral agents. American Journal of Epidemiology 2014; 159: 623-633.

37. Longini IM, et al. Containing pandemic influenza at the source. Science 2005; 309: 1083-1087.

38. Milne GJ, et al. A small community model for the transmission of infectious diseases: comparison of school closure as an intervention in individual-based models of an influenza pandemic. PLOS ONE. Published online: 23 December 2008. doi:10.1371/journal.pone.0004005.

39. Sander B, et al. Economic evaluation of influenza pandemic mitigation strategies in the United States using a stochastic microsimulation transmission model. Value in Health 2009; 12: 226-233.

40. van den Dool C, et al. The effects of influenza vaccination of health care workers in nursing homes: insights from a mathematical model. PLoS Medicine. Published online: 28 October 2009. doi:10.1371/journal. pmed.0050200.

41. Wu JT, et al. Hedging against antiviral resistance during the next influenza pandemic using small stockpiles of an alternative chemotherapy. PLoS Medicine. Published online: 19 May 2009. doi:10.1371/journal. pmed.1000085.

42. Dimitrov NB, et al. Optimizing tactics for use of the U.S. antiviral strategic national stockpile for pandemic influenza. PLoS ONE. Published online: 18 January 2011. doi:10.1371/journal.pone.0016094.

43. Lee VJ, Lye DC, Wilder-Smith A. Combination strategies for pandemic influenza response - a systematic review of mathematical modeling studies. BMC Medicine. Published online: 10 December 2009. doi:10.1186/ 1741-7015-7-76.

44. Prieto DM, et al. A systematic review to identify areas of enhancements of pandemic simulation models for operational use at provincial and local levels. BMC Public Health. Published online: 30 March 2012. doi:10.1186/ 1471-2458-12-251.

45. Reed C, et al. Novel framework for assessing epidemiologic effects of influenza epidemics and pandemics. Emerging Infectious Diseases 2013; 19: 85-91.
46. Riley $\mathbf{P}$, et al. Early characterization of the severity and transmissibility of pandemic influenza using clinical episode data from multiple populations. PLoS Computational Biology. Published online: 24 September 2015. doi: 10.1371/journal.pcbi.1004392.

47. World Health Organization. Pandemic influenza risk management: WHO interim guidance, 2013.

48. Ogunjimi B, et al. Using empirical social contact data to model person to person infectious disease transmission: an illustration for varicella. Mathematical Biosciences 2009; 218: 80-87.

49. Mossong J, et al. Social contacts and mixing patterns relevant to the spread of infectious diseases. PLoS Medicine. Published online: 25 March 2008. doi:10.1371/journal. pmed.0050074.

50. Aho M, et al. Outbreak of 2009 pandemic influenza A (H1N1) in a Finnish garrison - a serological survey. Eurosurveillance, 2010; 15, 19709.

51. Papenburg J, et al. Household transmission of the 2009 pandemic A/H1N1 influenza virus: elevated laboratoryconfirmed secondary attack rates and evidence of asymptomatic infections. Clinical Infectious Diseases, 2010; 51: 1033-1041.

52. Leung NHL, Xu C, Ip DKM, Cowling BJ. The fraction of influenza virus infections that are asymptomatic: a systematic review and meta-analysis. Epidemiology. Published online: 24 May 2015. doi:10.1097/EDE. 0000000000000340.

53. Diekmann O, Heesterbeek JAP, Metz JAJ. On the definition and the computation of the basic reproduction ratio $R_{0}$ in models for infectious diseases in heterogeneous populations. Journal of Mathematical Biology 1990; 28: 365-382.

54. Van Den Driessche $\mathbf{P}$, Watmough J. Reproduction numbers and sub-threshold endemic equilibria for compartmental models of disease transmission. Mathematical Biosciences, 2002; 180: 29-48.

55. Miller MR, et al. Predictors of influenza diagnosis among patients with laboratory-confirmed influenza. Journal of Infectious Diseases 2015; 212: 1604-1612.

56. Government of Alberta. Pandemic (H1N1) 2009: the Alberta experience (http://www.albertahealthservices.ca/ poph/hi-poph-surv-h1n1-ab-experience-2009.pdf). Accessed 6 February 2015.

57. Sikora C, et al. Transmission of pandemic influenza A (H1N1) 2009 within households: Edmonton, Canada. Journal of Clinical Virology, 2010; 49: 90-93.

58. Osterholm MT, et al. Efficacy and effectiveness of influenza vaccines: a systematic review and meta-analysis. Lancet 2012; 12: 36-44.

59. Tuite AR, et al. Estimated epidemiologic parameters and morbidity associated with pandemic h1n1 influenza. Canadian Medical Association Journal 2010; 182: 131-136.

60. Chowell G, Miller MA, Viboud C. Seasonal influenza in the United States, France, and Australia: transmission and prospects for control. Epidemiological Infection 2008; 136: 852-864.

61. Carrat F, et al. Time lines of infection and disease in human influenza: a review of volunteer challenge studies. American Journal of Epidemiology 2008; 167: 775-785. 
62. Killingley B, et al. Virus shedding and environmental deposition of novel $\mathrm{A}(\mathrm{H} 1 \mathrm{~N} 1)$ pandemic influenza virus: interim findings. Health Technology Assessment 2010; 14: 237-354.

63. Suess T, et al. Shedding and transmission of novel influenza virus $\mathrm{A} / \mathrm{H} 1 \mathrm{~N} 1$ infection in householdsGermany, 2009. American journal of epidemiology 2010; 171: 1157-1164.

64. Cao B, et al. Clinical features of the initial cases of 2009 pandemic influenza A (H1N1) virus infection in China. New England Journal of Medicine 2009; 361: 2507-2517.

65. Li IW, et al. The natural viral load profile of patients with pandemic 2009 influenza $\mathrm{A}(\mathrm{H} 1 \mathrm{~N} 1)$ and the effect of oseltamivir treatment. Chest Journal 2010; 137: 759-768.

66. Ling LM, et al. Effects of early oseltamivir therapy on viral shedding in 2009 pandemic influenza A (H1N1) virus infection. Clinical Infectious Diseases 2010; 50: 963-969.
67. Chen Y, et al. Risk factors for prolonged shedding of 2009 H1N1 influenza virus. Indian Pediatrics 2011; 48: 961-963.

68. Meschi S, et al. Duration of viral shedding in hospitalized patients infected with pandemic H1N1. BMC Infectious Diseases. Published online: 23 May 2011. doi:10.1186/1471-2334-11-140.

69. Na S, et al. Duration of viral shedding in patients admitted to hospital with pandemic influenza A/H1N1 2009 infection. Journal of Medical Virology 2011; 83: 5-9.

70. Siau C, et al. Influenza A H1N1 (2009): clinical spectrum of disease among adult patients admitted to a regional hospital in Singapore. Singapore Medical Journal 2011; 52: 475-480.

71. Biggerstaff $\mathbf{M}$, et al. Estimates of the reproduction number for seasonal, pandemic and zoonotic influenza: a systemic review of the literature. BMC Infectious Diseases. Published online: 4 September 2014. doi: 10.1186/1471-2334-14-480. 